\title{
Treatment of bone loss in proximal femurs of postmenopausal osteoporotic women with AGN1 local osteo-enhancement procedure (LOEP) increases hip bone mineral density and hip strength: a long-term prospective cohort study
}

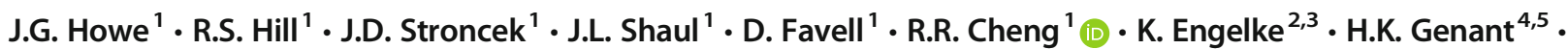 \\ D.C. Lee $^{6}$ - T.M. Keaveny ${ }^{7}$ - M.L. Bouxsein ${ }^{8}$ - B. Huber ${ }^{9}$
}

Received: 10 May 2019 / Accepted: 7 November 2019 / Published online: 4December 2019

(C) The Author(s) 2019

\begin{abstract}
Summary This first-in-human study of AGN1 LOEP demonstrated that this minimally-invasive treatment durably increased aBMD in femurs of osteoporotic postmenopausal women. AGN1 resorption was coupled with new bone formation by 12 weeks and that new bone was maintained for at least 5-7 years resulting in substantially increased FEA-estimated femoral strength. Introduction This first-in-human study evaluated feasibility, safety, and in vivo response to treating proximal femurs of postmenopausal osteoporotic women with a minimally-invasive local osteo-enhancement procedure (LOEP) to inject a resorbable triphasic osteoconductive implant material (AGN1).

Methods This prospective cohort study enrolled 12 postmenopausal osteoporotic (femoral neck T-score $\leq-2.5$ ) women aged 56 to 89 years. AGN1 LOEP was performed on left femurs; right femurs were untreated controls. Subjects were followed-up for 5-7 years. Outcomes included adverse events, proximal femur areal bone mineral density (aBMD), AGN1 resorption, and replacement with bone by X-ray and CT, and finite element analysis (FEA) estimated hip strength.

Results Baseline treated and control femoral neck aBMD was equivalent. Treated femoral neck aBMD increased by $68 \pm 22 \%$, $59 \pm 24 \%$, and $58 \pm 27 \%$ over control at 12 and 24 weeks and 5-7 years, respectively ( $p<0.001$, all time points). Using conservative assumptions, FEA-estimated femoral strength increased by $41 \%, 37 \%$, and $22 \%$ at 12 and 24 weeks and 5-7 years, respectively ( $p<0.01$, all time points). Qualitative analysis of X-ray and CT scans demonstrated that AGN1 resorption and replacement with bone was nearly complete by 24 weeks. By 5-7 years, AGN1 appeared to be fully resorbed and replaced with bone integrated with surrounding trabecular and cortical bone. No procedure- or device-related serious adverse events (SAEs) occurred.

Conclusions Treating femurs of postmenopausal osteoporotic women with AGN1 LOEP results in a rapid, durable increase in aBMD and femoral strength. These results support the use and further clinical study of this approach in osteoporotic patients at high risk of hip fracture.
\end{abstract}

Electronic supplementary material The online version of this article (https://doi.org/10.1007/s00198-019-05230-0) contains supplementary material, which is available to authorized users.

R.S. Hill

rhill@agnovos.com

AgNovos Healthcare LLC, Rockville, MD, USA

Bioclinica-Synarc, Inc., Hamburg, Germany

3 FAU University Erlangen-Nürnberg and Universitätsklinikum Erlangen, Erlangen, Germany

4 University of California San Francisco, San Francisco, CA, USA
5 Bioclinica-Synarc, Inc., Newark, CA, USA

6 O.N. Diagnostics, Berkeley, CA, USA

7 University of California Berkeley, Berkley, CA, USA

8 Beth Israel Deaconess Medical Center and Harvard Medical School, Boston, MA, USA

9 Mansfield Orthopedics, Morrisville, VT, USA 
Keywords Bone mineral density $\cdot$ Femoral strength $\cdot$ Finite element analysis $\cdot$ Hip fracture $\cdot$ Local osteo-enhancement procedure $\cdot$ LOEP · Osteoporosis · proximal femur

\section{Introduction}

Fragility fractures of the hip are associated with significant morbidity and mortality, and represent a significant burden on affected individuals, families, and healthcare systems [1-3]. Although multiple factors contribute to hip fracture risk, a key factor is osteoporosis, a disease characterized by bone loss $[4,5]$. By restoring bone loss due to osteoporosis, the weakened area is strengthened, resulting in increased force required to produce a fracture, thereby reducing fracture risk. Hip bone mineral density (BMD) is a major determinant of femoral bone strength explaining an estimated $70 \%$ of the strength [6]. It is particularly predictive of hip fracture risk versus fracture risk in other anatomical sites [7-9]. Other factors including age, family history, previous fractures, excessive drinking, visual acuity, falls, and dementia also play a role in determining fracture risk [10].

Despite current treatment options for osteoporosis, significant fracture risk remains, suggesting a need for new therapies [11]. Pharmacologic therapies that increase bone mass and reduce fracture risk in osteoporotic patients exist, but only a small proportion of patients are prescribed these therapies and when they are prescribed, compliance is low [12]. More importantly, existing therapies have not been shown to reduce fracture risk during the early stages of therapy, taking 9-18 months to significantly reduce hip fracture risk [11]. In patients with a recent hip fracture, the risk of a second contralateral hip fracture is significantly elevated in the months after the first fracture, and remains elevated for years [13]. There is a growing global consensus among leading clinicians that minimally-invasive surgical approaches intended to complement current therapies warrant consideration to further reduce risk of fragility fracture in patients at elevated risk $[11,14]$. The recently published guidance on diagnosis and management of postmenopausal osteoporosis in women by the International Osteoporosis Foundation (IOF) and European Society of Clinical and Economic Aspects of Osteoporosis (ESCEO) included a local osteo-enhancement procedure (LOEP) as a treatment option [15].

Prior investigations of surgical approaches to strengthen the proximal femur include the use of polymethyl methacrylate (PMMA) and prophylactic pinning or nailing [16, 17]. However, these non-biological approaches involve the permanent placement of inert materials that create a mismatch in elastic moduli with the surrounding bone, which may alter normal loadtransmission pathways.

In this study, we investigated the AGN1 local osteoenhancement procedure (LOEP), a minimally-invasive surgical approach to prepare an enhancement site, the area where new bone is desired within a local bony region weakened by osteoporotic bone loss, and to fill that site with a triphasic, resorbable, calcium-based implant material. This approach involves a single treatment that provides immediate strengthening [18], followed by resorption of the implant material and replacement with new bone.

This proof-of-concept study was designed to evaluate AGN1 LOEP treatment in postmenopausal women with osteoporosis, a population at elevated risk of fracture due to bone loss. The study was designed to determine: 1) the initial and long-term safety of treating the proximal femur using AGN1 LOEP, 2) the rate and extent of resorption and replacement of AGN1 with new bone in the proximal femur, and 3) initial and long-term changes in proximal femoral BMD and strength following AGN1 implantation.

\section{Methods}

A prospective, single-cohort study of AGN1 LOEP was conducted at a community-based hospital (Copley Hospital in Morrisville, VT, U.S.A.) between 2009 and 2016. Subjects were recruited from patients diagnosed with osteoporosis based on DXA scans completed at the Copley Hospital. Enrolled subjects were treated from 2009 to 2010 and were followed-up regularly for 24 months, with an additional follow-up visit 5-7 years after treatment in 2016, with an average follow-up of 6 years. The study was IRB-approved by the Western Institutional Review Board and all subjects provided written informed consent.

The study enrolled 12 Caucasian postmenopausal women. Inclusion criteria included age $\geq 55$ years and femoral neck aBMD T-score $\leq-2.5$ assessed by dual-energy X-ray absorptiometry (DXA, Hologic Delphi C). Note that one subject was admitted to the study with an aBMD T-score $>-2.5$. Subjects were excluded if they suffered a previous hip fracture, had creatinine $>2.0 \mathrm{mg} / 100 \mathrm{~mL}$, or a GFR $<30 \mathrm{~mL} / \mathrm{min}$. No additional blood analyses or biochemical markers were measured as part of the study. Subjects' pharmacological management of osteoporosis was not altered as a result of participating in the study. At enrollment, 6 subjects had been prescribed bisphosphonates and 1 subject had been prescribed hormone replacement therapy (Table 1). At the extension follow-up visit, 2 of the subjects had prescriptions for bisphosphonate treatment.

All study subjects received AGN1 LOEP treatment to the left proximal femur while no procedural treatment was performed on the right contralateral proximal femur, which served as a pairwise untreated control. All procedures were completed by the 
Table 1 Baseline demographic characteristics of study subjects

\begin{tabular}{ll}
\hline Characteristics & Baseline $(N=12)$ \\
\hline Age (years), mean (range) & $71.7(56-89)$ \\
Left femoral neck T-score, mean (SD) & $-2.9(0.4)$ \\
Right femoral neck T-score, mean (SD) & $-2.9(0.5)$ \\
Weight $(\mathrm{kg})$, mean (SD) & $53.5(10.6)$ \\
Height $(\mathrm{cm})$, mean (SD) & $157.2(5.4)$ \\
BMI $\left(\mathrm{kg} / \mathrm{m}^{2}\right)$, mean $(\mathrm{SD})$ & $21.6(3.9)$ \\
Current osteoporosis medication use, $N(\%)$ & \\
Bisphosphonate & $6(50 \%)$ \\
Hormone replacement therapy & $1(8 \%)$ \\
FRAX Calculated 10-year Risk ${ }^{*}$, mean $(\mathrm{range})$ & \\
Major osteoporotic fracture & $19.8 \%(9.8-38 \%)$ \\
Hip fracture & $8.6 \%(1.5-27 \%)$ \\
Comorbidities*, $N(\%)$ & \\
Gastroesophageal reflux disease & $5(42 \%)$ \\
Chronic obstructive pulmonary disease & $3(25 \%)$ \\
Depression & $3(25 \%)$ \\
Hypertension & $4(33 \%)$ \\
Hyperlipidemia & $3(25 \%)$ \\
Coronary artery disease & $1(8 \%)$ \\
Osteoarthritis & $6(50 \%)$ \\
Rheumatoid arthritis & $2(17 \%)$ \\
Parkinson's Disease & $1(8 \%)$ \\
Dementia & $1(8 \%)$ \\
\hline &
\end{tabular}

*Retrospectively assessed at extended follow-up visit.

two study investigators (BH, JGH), both orthopedic surgeons, at the Copley Hospital. All self-reported medical history data were verified through a medical record review.

AGN1 LOEP was performed with the subject positioned on a fracture table under anesthesia as recommended by the anesthesiologist. A $1 \mathrm{~cm}$ skin incision was made to gain access to the proximal lateral femoral cortex just below the greater trochanter, and a $2.5 \mathrm{~mm}$ guide pin was advanced centrally to the apex of the femoral neck under fluoroscopic guidance (Fig. 1a). A $5.3 \mathrm{~mm}$ cannulated drill was advanced over the guide pin to the subcapital femoral epiphyseal scar to access the enhancement site (Fig. 1b). The enhancement site was gently debrided and the site was irrigated with sterile saline and then aspirated to remove fat and other loose non-structural elements (Fig. 1c). The prepared AGN1 implant material was injected starting at the apex of the enhancement site (Fig. 1d) using low pressure under fluoroscopic guidance to fill the enhancement site. The injected AGN1, average implant volume $19 \pm 2 \mathrm{cc}$ (range 15 to $22 \mathrm{cc}$ ), completely sets within $60 \mathrm{~min}$ after mixing. Following recovery from anesthesia, subjects were fully weight-bearing within $4 \mathrm{~h}$ of the procedure. The skin incision healed rapidly in all subjects and the cortical access portal was healed in all 10 subjects as determined by $\mathrm{CT}$ imaging at the 5-7 year extension.
The study's primary outcome was comparison of the postinjection femoral neck areal bone mineral density (aBMD, $\mathrm{g} / \mathrm{cm}^{2}$ by DXA) versus the untreated contralateral femur with a secondary analysis comparing it to pre-treatment baseline values. aBMD values were equated to a T-score as appropriate using the NHANES 1998 reference values. DXA scans of both hips were taken at pre-treatment baseline and at 1,6 , 12,18 , and 24 weeks; 12,18 , and 24 months; and at the extension follow-up visit that occurred at an average of 6 years post-treatment. Extension aBMD was assessed by BioClinica. All other time points were assessed at Copley Hospital by a Hologic technician.

Anterior-posterior and lateral hip X-rays (pre-treatment, 6, 12 , and 24 weeks, and 6 years post-treatment) and bilateral hip CTs (pre-treatment, 12, and 24 weeks, CT Model Phillips Brilliance 16P scanner; and 6 years post-treatment, CT Model Siemens SOMATOM Definition AS scanner) were also acquired to qualitatively examine AGN1 resorption and to evaluate the extent of new bone formation.

For all subjects, axial and coronal planes and sometimes sagittal reformations of $\mathrm{CT}$ scans of all treatment visits were simultaneously viewed side-by-side. The approximate amount of high density (opaque) AGN1 remaining, in percentages, was judged from the marginated boundaries between the peripheral trabecular marrow space, an intermediate density zone of newly mineralized tissue and the higher density inner zone of unresorbed AGN1. All CT images were reviewed centrally on the same equipment to ensure consistency.

Femoral strength was estimated in simulated sideway fall loading condition using subject-specific nonlinear finite element analysis (FEA), as previously described [19]. Briefly, CT scans of the treated and untreated contralateral femurs were analyzed at pre-treatment, 12 and 24 weeks, and 6 years post-treatment using VirtuOst Version 1.2 (O.N. Diagnostics). VirtuOst is cleared by the FDA to measure bone strength, assess fracture risk, and monitor treatment effects, and has been shown to accurately measure bone strength [20]. Each finite element model consisted of eight-noded brick elements, $0.5 \mathrm{~mm}$ in length (approximately 1 million elements per model). Mechanical properties were derived from calibrated volumetric BMD values. The AGN1 volume of interest at the immediate post-operative time point was simulated using 12 -week images and registered to later time points. Within the implant region, a scale factor $(\alpha)$ was applied assuming either $100 \%$, or conservatively, $30 \%$ of the new tissue performed as normal load-bearing bone. As a conservative assumption, non-resorbed high-density implant material was assigned as minimal mechanical properties (Elastic modulus $=10 \mathrm{MPa}$; Poisson's Ratio $=0.3$; and Ultimate Strength $=0.1$ $\mathrm{MPa}$ ). Boundary conditions were applied with the diaphysis angled at $15^{\circ}$ with respect to the ground and $15^{\circ}$ of internal rotation to simulate an unprotected fall to the side of the hip. Femoral strength was defined from the non-linear force-strain 


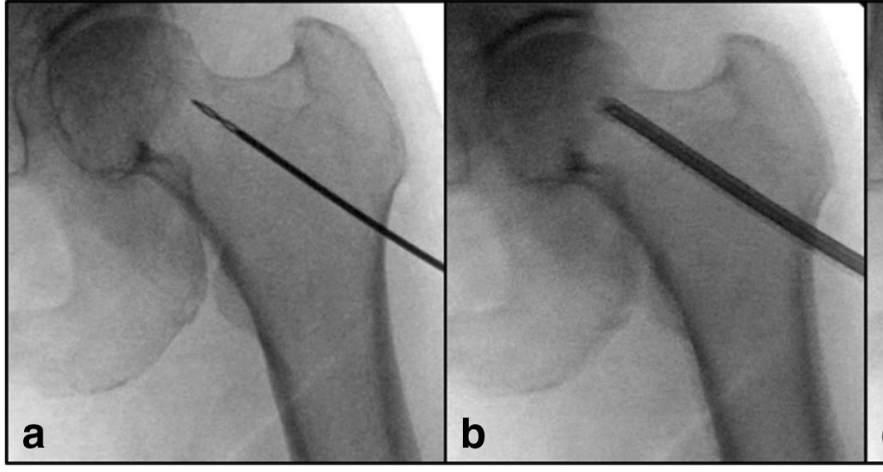

Fig. 1 Fluoroscopic series showing key steps in AGN1 LOEP. a Insertion of the guide pin to the apex of the femoral neck. b Cannulated drill advancement over the guide pin to the subcapital epiphyseal scar. c

curve as the force at $4.0 \%$ strain. Comparisons across all time points were limited to those subjects who had complete data from all three post-operative visits (9 subjects).

Statistical differences between treated and control hip quantitative measures were tested using paired T-tests at each time point.

Adverse events were evaluated and categorized according to standard definitions by the investigators with the aid of an independent study monitor. All adverse events were recorded at the time of the procedure, at each visit throughout the 24month follow-up period, and at the 6-year follow-up visit. An assessment of severity and relationship of causality to the device and/or the procedure was performed. Medical history, including the occurrence of any fragility fractures was recorded at each follow-up visit.

No subjects were lost to follow-up. All 12 subjects completed the 24-month follow-up visit and consented to the additional extended follow-up visit. Ten subjects were available for the imaging assessments at the extended follow-up visit. The two subjects not available for the imaging assessments completed medical history review.

\section{Results}

Subjects ranged in age from 56 to 89 years, with a mean of $71.7 \pm 10.1$ years (Table 1 ). Study subjects had low aBMD in the proximal femur and their left treated and right control hips did not differ in baseline aBMD (mean $0.527 \pm 0.054 \mathrm{~g} / \mathrm{cm}^{2}$ and $0.530 \pm 0.045 \mathrm{~g} / \mathrm{cm}^{2}$, respectively) or T-score (mean -2.9 \pm 0.4 ; range -2.2 to -3.4 and mean $-2.9 \pm 0.5$; range -2.3 to 4.1). Mean FRAX calculated 10-year risk of a hip fracture in these subjects at the onset of the study was $8.6 \%$ (range from 1.5 to $27 \%$ ). Subjects exhibited multiple comorbidities typical of this age group, i.e., osteoarthritis, gastroesophageal reflux disease, hypertension, and chronic obstructive pulmonary disease (Table 1).

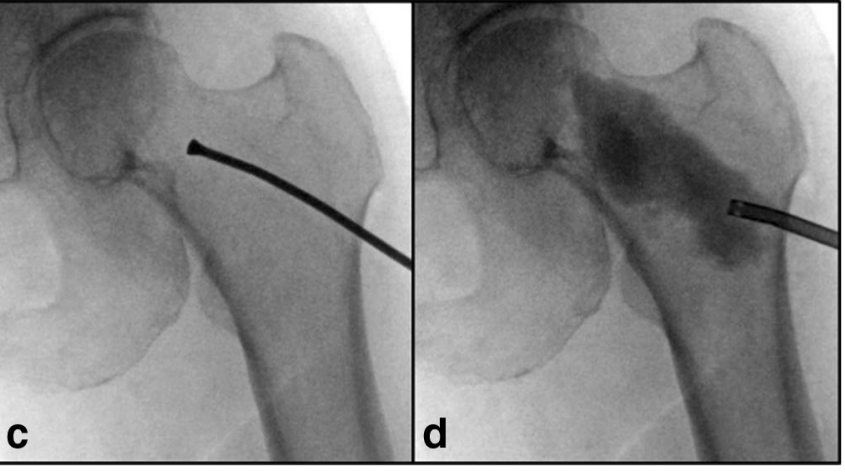

Gentle debridement to define the enhancement site followed by irrigation to remove loose elements. $\mathbf{d}$ Injection of the implant material to fill the enhancement site

Pre-treatment, X-ray (Fig. 2), and CT (Fig. 3) confirmed the scarcity of trabecular bone in the proximal femur at baseline. In all subjects, X-rays showed that the AGN1 implant material within the intended enhancement site remained where it was implanted in the proximal femur until it was resorbed and replaced with bone (Fig. 2). AGN1's initial uniform high density radiopacity concentrically changed to an intermediate density as AGN1 resorbed. This change in size and density was evident in both X-ray (Figure 2) and CT (Fig. 3) imaging as AGN1 was replaced with newly formed bone. In the proximal femur, this newly formed bone was remodeled and was still evident at the 5-7 year extension. In contrast, AGN1 implant material that was initially within the medullary canal of the femur of some subjects was resorbed and replaced with mineralized tissue. However, by the 5-7 year follow-up, this more distal mineralized tissue was no longer present (e.g., Subject 11, Fig. 3). X-ray analysis demonstrated that AGN1 was completely resorbed in 4 subjects by 24 weeks post-treatment, and in all 10 subjects imaged at the 5-7 year extension. CT confirmed these results. By 24 weeks, semi-quantitative analysis of CT images demonstrated that only $17 \pm 14 \%$ of AGN1 remained with complete resorption by the extension time point (see Supplemental Data Table 1).

All 12 subjects demonstrated a large immediate increase in femoral neck aBMD at 1 week after AGN1 injection (Fig. 4). This immediate increase remained significantly greater than the corresponding control hips at all subsequent time points ( $p$ $<0.0001)$. Specifically, the treated femoral neck aBMD was $68 \pm 22 \%$ higher than the control at 12 months; $59 \pm 24 \%$ greater at 24 months; and $58 \pm 27 \%$ higher at $5-7$ years posttreatment. From 1 to 24 weeks after treatment, there was a rapid decrease in aBMD, which coincided with AGN1 resorption. After 24 weeks, there was a slower decrease in aBMD that remained significantly elevated above untreated hips at all individual time points through the 5-7 year follow-up. Although not significant, femoral neck aBMD declined $1.2 \%$ and $1.4 \%$ from 24 months until the extended followup visit in the treated hips and control hips, respectively. 


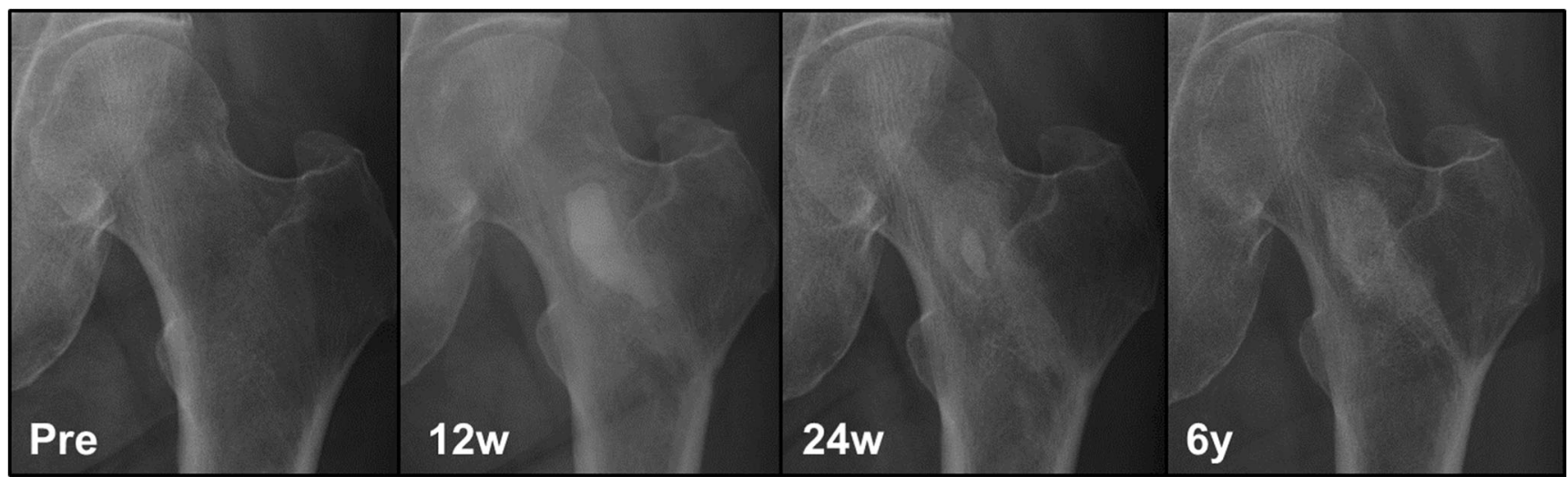

Fig. 2 Representative X-ray series from subject 07 showing AGN1 resorption and replacement with new bone in the proximal femur; left to right: pretreatment, post-treatment 12 weeks, 24 weeks, and 311 weeks

A consistent response was observed in all subjects. All regions of the proximal femur showed a similar temporal pattern of aBMD change as seen in the femoral neck, i.e., an immediate dramatic increase, a period of rapid decrease as AGN1 resorbed, followed by a gradually declining steady state that remained significantly greater than the control to the final time point. The increase in aBMD following AGN1 LOEP treatment was the same in patients who were or were not prescribed with antiresorptive therapy. Subject compliance with medication, however, was not captured. At no time point in any subject did the treated hip aBMD in any region drop below that of the control hip (Supplemental Data Tables 2 to 13).
All subjects exhibited large increase in femoral strength on the treated side, as assessed by FEA simulating a sideways fall configuration. Two sets of results are presented to provide bounds on the expected in vivo behavior accounting for the range in the biomechanical properties of the newly formed mineralized tissue in the originally implanted region. Within the implant region, a scale factor $(\alpha)$ was applied assuming either $100 \%$, or conservatively, $30 \%$ of the new tissue performed as normal load-bearing bone. Regardless of the scale factor used, femoral strength was significantly higher in the treated compared with the contralateral control femur at 12 weeks, 24 weeks, and 5-7 years following the LOEP
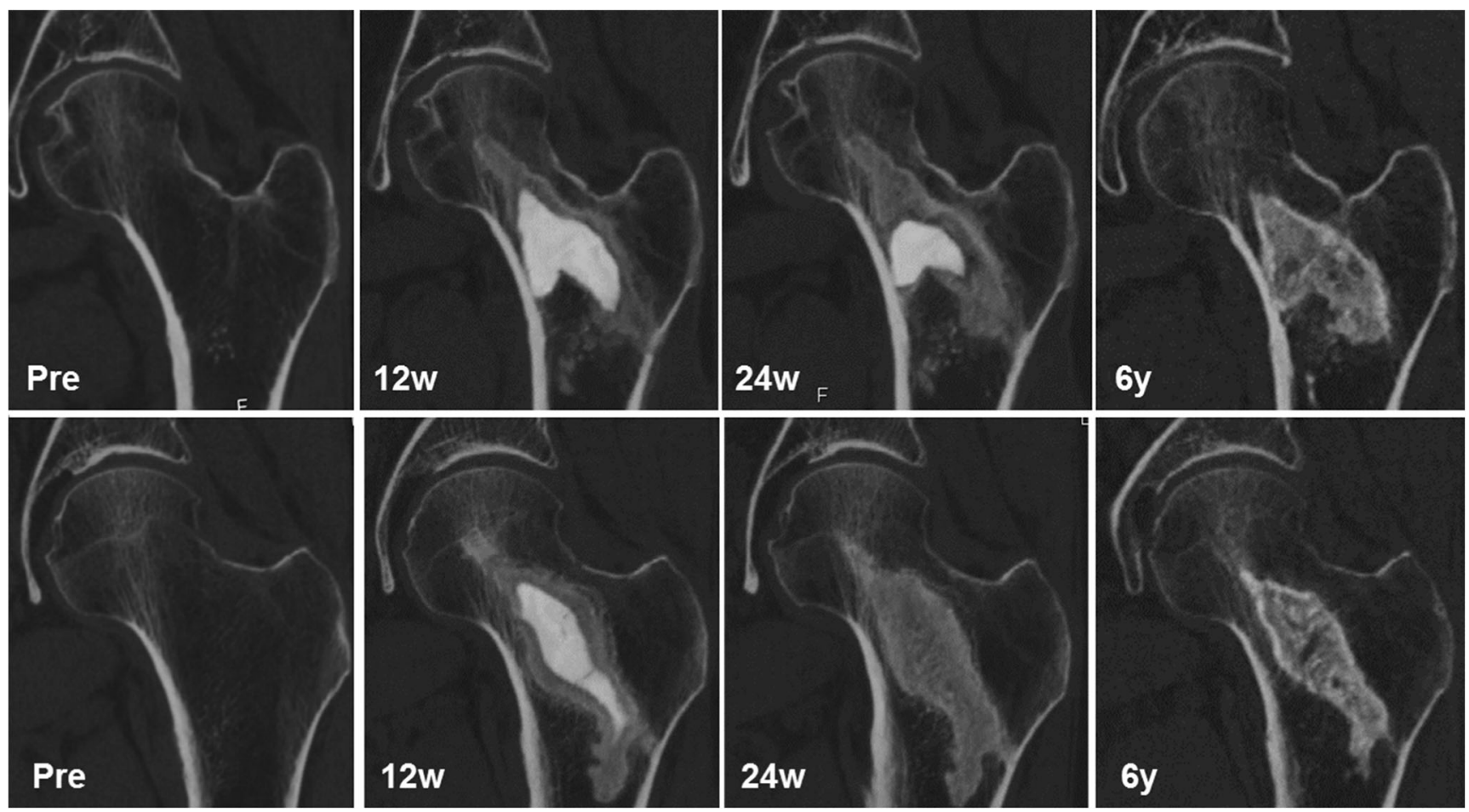

Fig. 3 CT series from subject 06 (top row) and Subject 11 (bottom row) showing paucity of trabeculae in the femoral neck at baseline, and subsequent formation and remodeling of new bone; left to right: Pre-treatment, Post-treatment 12 weeks, 24 weeks, and at the extension follow-up average of 6 years 


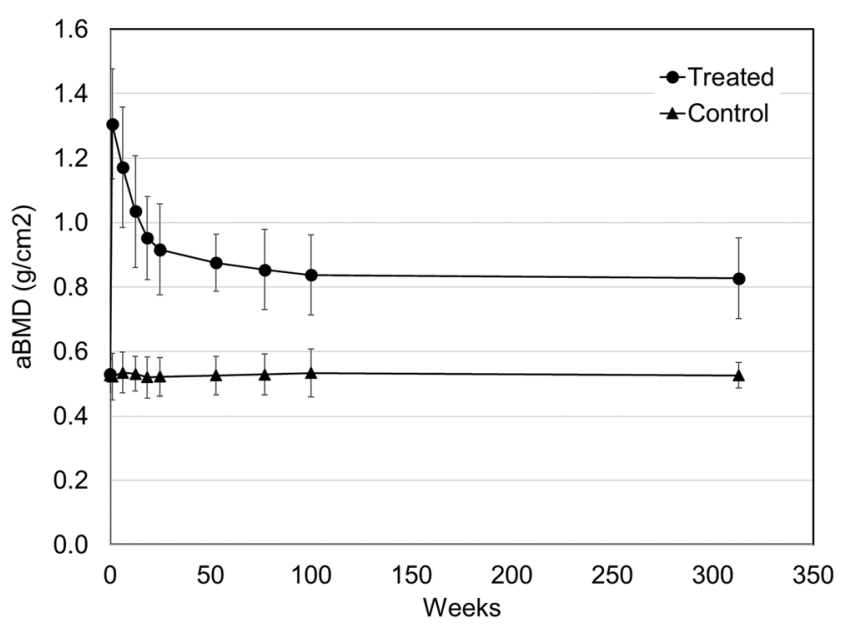

Fig. 4 Femoral neck aBMD in treated and control hips as assessed by DXA. $N=12$ except at 315 weeks $N=10 ; p<0.001$ treated vs control for all time points

procedure $(p<0.01$; Table 2$)$. At 12 weeks, hip strength was on average higher in the treated versus control hip by $59 \%(\alpha$ $=1.00)$ or $41 \%(\alpha=0.30)(p<0.01)$. At 24 weeks, the average hip strength was $54 \%(\alpha=1.00)$ or $37 \%(\alpha=0.30)(p<0.01)$ higher in the treated than control hip. At 5-7 years, the treatment effect persisted as the strength of the treated femurs was $36 \%(\alpha=1.00)$ or $22 \%(\alpha=0.30)(p<0.01)$ higher relative to control femurs.

There were no procedure or device-related serious adverse events and there were also no unanticipated adverse events. Ten adverse events were reported in five subjects, none of which were device-related (pneumonia, shoulder pain, and squamous cell carcinoma). Three adverse events were at least possibly related to the LOEP surgical procedure (small area of wound breakdown, irritation from the injection procedure, and post-operative nausea), which were mild and resolved without additional medical intervention. Seven osteoporosis-related fragility fractures were observed: 2 control hips ( 27 and 44 months), 1 treated hip (40 months), 1 patella (8 months), 2 vertebrae (73 months and unknown), and 1 humerus (35 months)

Table 2 FEA-estimated femoral strength in sideways fall

\begin{tabular}{llll}
\hline Time point & Control femur strength $(N)$ & $\begin{array}{l}\text { Treated femur } \\
\text { strength }(N)\end{array}$ \\
\hline Pre-treatment & $2028 \pm 469$ & $2077 \pm 469$ & \\
Post-treatment & & $\alpha=0.30^{\mathrm{a}}$ & $\alpha=1.00^{\mathrm{a}}$ \\
12 Weeks & $1994 \pm 425$ & $2820 \pm 463^{*}$ & $3165 \pm 432^{*}$ \\
24 Weeks & $2013 \pm 425$ & $2755 \pm 402^{*}$ & $3101 \pm 392^{*}$ \\
315 Weeks & $1981 \pm 338$ & $2420 \pm 396^{*}$ & $2685 \pm 403^{*}$
\end{tabular}

${ }^{\text {a }}$ Scale factor $(\alpha)$ was applied to implant region assuming either $30 \%$ or $100 \%$ of the new tissue performed as normal load-bearing bone. Statistical significance as assessed by paired $t$ test indicated with *indicates $p<0.01$ when compared with the control at the same time point.
(Table 3). One vertebral fracture at 12 months posttreatment resulted from a motor vehicle accident was not classified as a fragility fracture.

It is unlikely that detection bias contributed to the results given no subjects were lost to follow-up and a review of medical charts for all subjects to verify data was completed.

\section{Discussion}

This first-in-human study examined the potential utility of a novel local osteo-enhancement procedure to improve hip bone strength in postmenopausal women. A unique aspect of AGN1 LOEP treatment is that it directly addresses osteoporotic bone loss in the area treated. Moreover, AGN1 implant material placement was similar to the optimal placement within the femoral neck and intertrochanteric region as outlined by Basafa et al. [21]. Accordingly, treatment with AGN1 LOEP resulted in notable, statistically significant, and sustained increases in proximal femur aBMD and these changes in aBMD correlated with a substantial increase in femoral strength. In particular, femoral neck aBMD in the treated hip was on average $58 \%$ greater and femoral strength was $36 \%$ greater than the control hip at 5-7 years after treatment.

The time course of aBMD changes following LOEP can be explained by the nature of the AGN1 material and its resorption profile. Notably, the AGN1 implant material has a high radiopacity, thus explaining the immediate and large femoral neck aBMD increase immediately after treatment. Thereafter, as seen in the X-ray and CT analyses, the AGN1 is rapidly resorbed, leading to a notable decrease in aBMD over the first 24 weeks. However, by 24 weeks AGN1 was substantially resorbed and residual AGN1 contributed only minimally to aBMD. AGN1 was completely resorbed and made no contribution to aBMD from 1-year after treatment through the 5-7 year follow-up. Therefore, the sustained long-term increases in aBMD and femoral strength were due to newly formed bone and not to residual AGN1.

AGN1 resorption was closely coupled to new bone formation and the area of bone formation correlated with the area of AGN1 implantation, i.e., the enhancement site of AGN1 injection appeared to provide a template for bone formation. A consistent treatment response was observed in all subjects, with no discernable difference among subjects regarding AGN1 resorption, aBMD and femoral strength changes after AGN1 treatment, or changes in X-ray or CT images, despite variable use of osteoporosis pharmacologic therapies by these subjects. The consistency and magnitude of the increases in femoral strength combined with the sustained nature of the femoral strength gains through 5-7 years indicate that AGN1 LOEP likely provides a long-term benefit to osteoporotic subjects. In addition to the likely long-term benefit, a prior study in human cadaveric femurs demonstrated that 
Table 3 Summary of all fragility fractures

\begin{tabular}{lllll}
\hline Subject & Fracture location & Time post-procedure & Age at fracture & Cause \\
\hline 02 & Left proximal humerus & 36 months & 83 & Fall \\
03 & Left patella & 8 months & 87 & Fall \\
& Left hip (treated) & 40 months & 90 & Unknown \\
& Right hip (control) & 44 months & 90 & Fall \\
& Spine (level unknown) & 73 months & 93 & Unknown \\
05 & Right hip (control) & 27 months & 67 & Fall \\
08 & Spine (T8) & Unknown & Unknown & Unknown \\
\hline
\end{tabular}

AGN1 implantation also leads to an immediate increase in femoral strength [18]. Although the sample size does not allow for a statistical assessment of fracture reduction, increased aBMD and femoral strength via FEA correlates with bone strength and fracture risk reduction [19, 20, 22, 23], suggesting that AGN1 LOEP has the potential to reduce hip fracture risk.

Several lines of evidence indicate that the newly formed bone is functional with the characteristics necessary to reduce the risk of hip fracture by increasing the force needed to fracture. First, the $1.2 \%$ decline in aBMD in the treated hip from the 24-month to the 5-7-year follow-up is what one would expect of ongoing bone turnover in these osteoporotic patients, i.e., the new bone is responding as one would expect of existing bone in these patients. This observation is consistent with the assertion that increased density in the implant area is due to newly formed bone that exhibits features of normal bone metabolism. Second, both the X-ray and CT analyses suggest that the newly formed bone matured and became integrated and incorporated into the surrounding trabecular bone. This observation of bone maturation and enhancement of structural integrity of the proximal femur (Fig. 3) is supported by the absence of radiolucency surrounding the area of new bone formation as well as the presence of larger trabecular plates extending to and from the newly formed bone and adjacent cortical bone. Third, the evolution of the radiographic appearance of the newly formed bone through the last time point suggests biologic transformation of the bone through the final follow-up time point. This is seen in density increases in portions of the outer border of the implanted area related to bone formation, while there was some diminution in the overall size of the denser area from the 24-week to the average 6-year followup interval. Note the 6-year CT images were performed on a different CT scanner with higher spatial resolution (Fig. 3). Fourth, newly formed bone tended to remain in areas that were under mechanical stress, while resorbing from areas of minimal stress (Fig.3), such as the medullary canal, again consistent with normal bone metabolism. Finally, the subject-specific FEA demonstrated substantial improvement in femoral strength at all follow-up time points.
There is ample evidence for a strong correlation between aBMD of the proximal femur and hip fracture risk [8, 22]. For example, in the HORIZON Recurrent Fracture Trial, which tested whether zoledronic acid would prevent contralateral hip fractures in those who had already suffered one hip fracture, modest increases in femoral neck aBMD (3.6\% over 3 years) led to a clinically meaningful and significant $30 \%$ decrease in the incidence of hip fractures [24]. Similarly, strong correlations have been demonstrated between FEA-estimated hip strength and hip fracture risk [19, 20]. For example, denosumab treatment increased FEA-estimated hip strength $9 \%$ relative to baseline at 36 months [25], which corresponded to an observed hip fracture reduction of $42 \%$ [26]. Comparatively speaking, AGN1 LOEP treatment resulted in a sustained $58 \%$ average BMD increase and $36 \%$ increase in FEA-estimated hip strength after 5-7 years. These results suggest that AGN1 LOEP has the potential to dramatically reduce fracture risk with a single treatment.

Hip fracture risk reduction with existing drug therapies is achieved only when patients are compliant and persistent with treatment. However, less than $35 \%$ of patients continue with osteoporosis therapy at 1 year [12, 27]. Real-world hip fracture prevention with drug therapy is further limited due to under-diagnosis of osteoporosis, limited prescription of pharmaceutical therapies to osteoporotic patients, delayed onset of effectiveness of these therapies, and an incomplete 30 to $51 \%$ hip fracture risk reduction [11, 24, 26, 28]. In contrast, AGN1 LOEP provides immediate [18] and as noted above, long-term protection, and compliance is guaranteed as the treatment is a one-time procedure. Moreover, the AGN1 LOEP treatment response was observed in all subjects whether or not they were taking anti-resorptive drugs, demonstrating the feasibility of using AGN1 LOEP in patients on systemic osteoporosis therapy.

Like osteoporosis medications and other nonpharmacologic means of managing hip fracture risk (i.e., lifestyle changes), the AGN1 LOEP treatment is expected to significantly reduce, but not eliminate, the risk of fragility hip fractures. Two control hips ( 2 of $12,16.6 \%)$ and one treated hip ( 1 of $12,8.3 \%$ ) fractured during the 72 patients' years of follow-up (Table 3). The fracture of the AGN1 LOEP treated 
femur was a subcapital fracture that was successfully repaired by hemiarthroplasty without complication. The treated hip fracture occurred in Subject 03 (Table 3) with the most fragility fractures and the greatest number of risk factors for a hip fracture including advanced age (90 years), Parkinson's disease, dementia, stroke, and COPD.

Past efforts to locally strengthen the proximal femur, and thereby reduce the risk of hip fracture, generally focused on the implantation of inert, permanent materials (e.g. PMMA) $[16,29-32]$. In contrast, this is a biological approach to locally strengthen the proximal femur by replacing bone lost due to osteoporosis. As a single-center 12 subject study, the experience and conclusions from this study may not be generalizable to the broader population; however, the results are promising and support the potential clinical utility of this new treatment. The safety profile of this treatment observed in this study is consistent with other minimally-invasive orthopedic procedures (e.g., femoral head core decompression). This study supports the safety of this minimally-invasive surgical intervention as a new approach to treat osteoporotic bone loss and reduce hip fracture risk.

In conclusion, this first-in-human study establishes AGN1 LOEP as an emerging treatment for local osteoporotic bone loss in patients at high risk of hip fracture and provides strong rationale for further clinical investigation of this treatment.

Acknowledgments The study investigators and authors are indebted to the subjects who consented to participate in the study and to all participating staff at the investigational center.

Funding information The study was sponsored by AgNovos.

\section{Compliance with ethical standards}

Conflicts of interest JGH is the founder and a shareholder of AgNovos. RSH, JDS, JLS, DF, and RRC are/were employees and shareholders of AgNovos. HKG has received honoraria, consulting fees, and speaker fees from AgNovos, Medtronic, Regeneron, BioMarin, Clementia, Amgen, Lilly, Daiichi, AstraZeneca, and MedImmune. MB has received consulting fees from AgNovos and research funding from Radius and Amgen. DCL is an employee of and has equity interest in O.N. Diagnostics. TMK is the founder, shareholder, and consulting Chief Science Officer of O.N. Diagnostics and has received consulting fees from AgNovos, O.N. Diagnostics, and Amgen. BH has received consulting fees from and is a shareholder of AgNovos.

Ethical approval The study was IRB-approved by the Western Institutional Review Board and all subjects provided written informed consent.

Open Access This article is distributed under the terms of the Creative Commons Attribution-NonCommercial 4.0 International License (http:// creativecommons.org/licenses/by-nc/4.0/), which permits any noncommercial use, distribution, and reproduction in any medium, provided you give appropriate credit to the original author(s) and the source, provide a link to the Creative Commons license, and indicate if changes were made.

\section{References}

1. Johnell O, Kanis JA (2004) An estimate of the worldwide prevalence, mortality and disability associated with hip fracture. Osteoporos Int 15:897-902. https://doi.org/10.1007/s00198-0041627-0

2. Burge R, Dawson-Hughes B, Solomon DH, Wong JB, King A, Tosteson A (2007) Incidence and economic burden of osteoporosis-related fractures in the United States, 2005-2025. J Bone Miner Res 22:465-475. https://doi.org/10.1359/jbmr.061113

3. Abrahamsen B, Van Staa T, Ariely R et al (2009) Excess mortality following hip fracture: a systematic epidemiological review. Osteoporos Int 20:1633-1650. https://doi.org/10.1007/s00198009-0920-3

4. Kanis JA (1994) Assessment of fracture risk and its application to screening for postmenopausal osteoporosis: synopsis of a WHO report. Osteoporos Int 4:368-381. https://doi.org/10.1007/ BF01622200

5. Kanis JA, Oden A, Johansson H et al (2009) FRAX® and its applications to clinical practice. Bone 44:734-743. https://doi.org/10. 1016/j.bone.2009.01.373

6. Marshall D, Johnell O, Wedel H (1996) Meta-analysis of how well measures of bone mineral density predict occurrence of osteoporotic fractures. BMJ 312:1254-1259. https://doi.org/10.1136/bmj.312. 7041.1254

7. Stone KL, Seeley DG, Lui L-Y et al (2003) BMD at multiple sites and risk of fracture of multiple types: long-term results from the study of osteoporotic fractures. JBMR 18:1047-1954. https://doi. org/10.1359/jbmr.2003.18.11.1947

8. Johnell O, Kanis JA, Oden A, Johansson H, de Laet C, Delmas P, Eisman JA, Fujiwara S, Kroger H, Mellstrom D, Meunier PJ, Melton LJ 3rd, O'Neill T, Pols H, Reeve J, Silman A, Tenenhouse A (2005) Predictive value of BMD for hip and other fractures. J Bone Miner Res 20:1185-1194. https://doi.org/10.1359/JBMR. 050304

9. Melton LJ, Riggs BL, Keaveny TM et al (2007) Structural determinants of vertebral fracture risk. J Bone Miner Res 22:1885-1892. https://doi.org/10.1359/jbmr.070728

10. Marks R (2010) Hip fracture epidemiological trends, outcomes, and risk factors background to the problem. Int J Gen Med 3:1-17. https://doi.org/10.2147/JJGM.S5906

11. Ferrari S, Reginster J-Y, Brandi ML, Kanis JA, Devogelaer JP, Kaufman JM, Féron JM, Kurth A, Rizzoli R (2016) Unmet needs and current and future approaches for osteoporotic patients at high risk of hip fracture. Arch Osteoporos 11:37-12. https://doi.org/10. 1007/s11657-016-0292-1

12. Silverman SL, Schousboe JT, Gold DT (2011) Oral bisphosphonate compliance and persistence: a matter of choice? Osteoporos Int 22: 21-26. https://doi.org/10.1007/s00198-010-1274-6

13. Ryg J, Rejnmark L, Overgaard S et al (2009) Hip fracture patients at risk of second hip fracture: a nationwide population-based cohort study of 169,145 cases during 1977-2001. J Bone Miner Res 24: 1299-1307. https://doi.org/10.1359/jbmr.090207

14. Varga P, Hofmann-Fliri L, Blauth M, Windolf M (2016) Prophylactic augmentation of the osteoporotic proximal femurmission impossible? Bonekey Rep 5:1-33. https://doi.org/10.1038/ bonekey.2016.86

15. Kanis JA, Cooper C, Rizzoli R, Reginster J-Y (2018) European guidance for the diagnosis and management of osteoporosis in postmenopausal women. Osteoporos Int. https://doi.org/10.1007/ s00198-018-4704-5

16. Beckmann J, Springorum R, Vettorazzi E, Bachmeier S, Lüring C, Tingart M, Püschel K, Stark O, Grifka J, Gehrke T, Amling M, Gebauer M (2011) Fracture prevention by femoroplasty-cement 
augmentation of the proximal femur. J Orthop Res 29:1753-1758. https://doi.org/10.1002/jor.21410

17. Szpalski M, Gunzburg R, Aebi M et al (2015) A new approach to prevent contralateral hip fracture: evaluation of the effectiveness of a fracture preventing implant. Clin Biomech 30:713-719. https:// doi.org/10.1016/j.clinbiomech.2015.05.001

18. Stroncek JD, Shaul JL, Favell D, Hill RS, Huber BM, Howe JG, Bouxsein ML (2019) In vitro injection of osteoporotic cadaveric femurs with a triphasic calcium-based implant confers immediate biomechanical integrity. J Orthop Res 37:908-915. https://doi.org/ 10.1002/jor.24239

19. Kopperdahl DL, Aspelund T, Hoffmann PF, Sigurdsson S, Siggeirsdottir K, Harris TB, Gudnason V, Keaveny TM (2014) Assessment of incident spine and hip fractures in women and men using finite element analysis of CT scans. J Bone Miner Res 29:570-580. https://doi.org/10.1002/jbmr.2069

20. Johannesdottir F, Thrall E, Muller J et al (2017) Comparison of noninvasive assessments of strength of the proximal femur. Bone 105: 93-102. https://doi.org/10.1016/j.bone.2017.07.023

21. Basafa E, Murphy RJ, Otake Y et al (2015) Subject-specific planning of femoroplasty: an experimental verification study. J Biomech 48:59-64. https://doi.org/10.1016/j.jbiomech.2014.11.002

22. Bouxsein ML, Eastell R, Lui L-Y, Wu LA, de Papp AE, Grauer A, Marin F, Cauley JA, Bauer DC, Black DM, FNIH Bone Quality Project (2019) Change in bone density and reduction in fracture risk: a meta-regression of published trials. J Bone Miner Res 34: 632-642. https://doi.org/10.1002/jbmr.3641

23. Lee DC, Varela A, Kostenuik PJ, Ominsky MS, Keaveny TM (2016) Finite element analysis of denosumab treatment effects on vertebral strength in ovariectomized cynomolgus monkeys. J Bone Miner Res 31:1586-1595. https://doi.org/10.1002/jbmr.2830

24. Lyles KW, Colón-Emeric CS, Magaziner JS et al (2007) Zoledronic acid and clinical fractures and mortality after hip fracture. $\mathrm{N}$ Engl $\mathrm{J}$ Med 357:1799-1809. https://doi.org/10.1056/NEJMoa074941

25. Keaveny TM, McClung MR, Genant HK, Zanchetta JR, Kendler D, Brown JP, Goemaere S, Recknor C, Brandi ML, Eastell R, Kopperdahl DL, Engelke K, Fuerst T, Radcliffe HS, Libanati C
(2014) Femoral and vertebral strength improvements in postmenopausal women with osteoporosis treated with denosumab. J Bone Miner Res 29:158-165. https://doi.org/10.1002/jbmr.2024

26. Cummings SR, Martin JS, McClung MR et al (2009) Denosumab for prevention of fractures in postmenopausal women with osteoporosis. N Engl J Med 361:756-765. https://doi.org/10.1056/ NEJMoa0809493

27. Solomon DH, Johnston SS, Boytsov NN, McMorrow D, Lane JM, Krohn KD (2014) Osteoporosis medication use after hip fracture in U.S. patients between 2002 and 2011. J Bone Miner Res 29:1929 1937. https://doi.org/10.1002/jbmr.2202

28. McClung MR, Geusens P, Miller PD, Zippel H, Bensen WG, Roux C, Adami S, Fogelman I, Diamond T, Eastell R, Meunier PJ, Reginster JY, Hip Intervention Program Study Group (2001) Effect of risedronate on the risk of hip fracture in elderly women. N Engl J Med 344:333-340

29. Heini PF, Franz T, Fankhauser C, Gasser B, Ganz R (2004) Femoroplasty-augmentation of mechanical properties in the osteoporotic proximal femur: a biomechanical investigation of PMMA reinforcement in cadaver bones. Clin Biomech 19:506-512. https:// doi.org/10.1016/j.clinbiomech.2004.01.014

30. Sutter EG, Mears SC, Belkoff SM (2010) A biomechanical evaluation of femoroplasty under simulated fall conditions. J Orthop Trauma 24:95-99. https://doi.org/10.1097/BOT. 0b013e3181b5c0c6

31. Sutter EG, Wall SJ, Mears SC, Belkoff SM (2010) The effect of cement placement on augmentation of the osteoporotic proximal femur. Geriatr Orthop Surg Rehabil 1:22-26. https://doi.org/10. 1177/2151458510378406

32. Fliri L, Sermon A, Wähnert D et al (2013) Limited V-shaped cement augmentation of the proximal femur to prevent secondary hip fractures. J Biomater Appl 28:136-143. https://doi.org/10.1177/ 0885328212443274

Publisher's note Springer Nature remains neutral with regard to jurisdictional claims in published maps and institutional affiliations. 